\title{
Flexible synthetic mobility modeling to discover trajectories for complex areas of mobile wireless networks
}

\author{
Nisrine Ibadah · Mohammed Rziza · Khalid Minaoui · Mohammed Oumsis · César Benavente-Peces
}

\begin{abstract}
Mobility modeling is one of the most influential pillars in improving the performance of wireless networks. Understanding mobility features is relevant for the design and analysis of proper motion schemes for any network. Up to now, a variety of entity mobility models have been suggested which do not take into account all the characteristics of real-life movements (time, space, environment). In order to obtain an improved model that overcomes such limitations, in this paper a new hybrid synthetic entity mobility model is proposed, called the Maze mobility model (MzMM). It takes into consideration the timeline history, spatial dependencies, and geographical restrictions as well. At the same time, it respects the laws of motion to reflect actual scenarios using a flexible discovery mechanism, where it permits nodes arriving at predefined destinations following the most appropriate trajectories in the presence of several obstacles. This approach allows mobile nodes to move correctly even in the presence of multiple mobility constraints. The significance of this research is that the new approach considers a realistic combination of parameters to achieve a flexible and robust mobility model that can be applied for autonomous or human mobility, even in complex environments to provide optimized performances for networks, as demonstrated by its high performance results.
\end{abstract}

Keywords Maze mobility model $\cdot$ Mobility model $\cdot$ Routing protocol $\cdot$ Performance analysis $\cdot$ Wireless network

\begin{tabular}{|c|c|c|}
\hline & \multicolumn{2}{|c|}{ Abbreviations } \\
\hline & MzMM & Maze mobility model \\
\hline & QoS & Quality of service \\
\hline & MMs & Mobility models \\
\hline & MANET & Mobile ad hoc network \\
\hline & VANET & Vehicular ad-hoc network \\
\hline & WSN & Wireless sensor network \\
\hline & RWMM & RandomWaypoint MM \\
\hline & MGMM & Manhattan grid mobility model \\
\hline & RPGMM & Reference point group MM \\
\hline & NMM & Nomadic MM \\
\hline & SLAW & Self-similar least action walk \\
\hline & AODV & Ad hoc on-demand distance vector \\
\hline & OLSR & Optimized link state routing protocol \\
\hline & ZRP & Zone routing protocol \\
\hline & PDR & Packet delivery ratio \\
\hline & RREQ & Route request \\
\hline 1 & RREP & Route reply \\
\hline & IARP & Intra-zone routing protocol \\
\hline & IERP & Inter-zone routing protocol \\
\hline
\end{tabular}




\section{Introduction}

Wireless communications have experienced an unexpected growth in their data traffic, as mobile applications and services have become omnipresent in our daily life. Nowadays, a wide diversity of mobile devices can be interconnected, due mainly to successful technological advances. In consequence, challenging issues requiring more efficient and novel protocols and services without communication gaps and offering a high quality of service (QoS) are constantly being addressed by both academia and industry. The achievement of appropriate mobility models is one of the most impacting developments to improve the performance of wireless networks. The relevance of mobility models relays on the fact that they are used for simulating and evaluating mobile wireless networks overall performance and to design and assess algorithms and protocols which are based on them. Frequent mobility represents one of those challenging issues where it is deeply examined by considering highly dynamic mobile networks, leading the network mobility models to aim at characterizing the nodes movement (Tomasini et al. 2017; Ibadah et al. 2017). Several researchers have demonstrated that the adopted motion model significantly impacts the whole network performance (Karamshuk et al. 2011). Determining an appropriate mobility model allows increasing the overall network performance, as it helps the routing protocols to succeed. For instance, routing protocols are heavily influenced by node mobility, where the same protocol performs in a completely different way for different motions of the nodes. Many research has been carried out in which the mobility model is the focus of attention. An emerging topic concerns mobility models for opportunistic networks (Conti and Giordano 2014), including the Mobile Ad Hoc Network (MANET) (Cheng et al. 2013) and Vehicular Ad-hoc Networks (VANET), where node mobility is used to achieve message delivery (Da Cunha et al. 2014). An important area of research focuses on mobility modeling to shape different designs with distinct mobility requirements in order to somehow reflect real-life movements. Various mobility models (MMs) are mainly applied to the ground, space, airborne, and undersea, where devices are mobile. These models are proposed to represent mobility decisions which must be taken into consideration to describe the relations between speed, time, and distance. The performance of mobile wireless networks is remarkably impacted by the mobility model adopted. These models are proposed to represent mobility decisions which must be taken into consideration to describe the relations between speed, time, and distance. Nevertheless, in complex environments, i.e., in the presence of many constraints, a simple pattern cannot properly guide a given mobile node to reach a predefined destination and, hence, it doesn't obtain the best performance outcomes.

Nevertheless, this field of research is constantly eager to experiment and implement new models. Our proposed mobility model combines all the aforementioned subclasses. Based on an analysis of the state of the art of patterns which have been previously suggested (Batabyal and Bhaumik 2012), we propose a new hybrid entity synthetic mobility model called the Maze mobility model (MzMM) to deal with current and arising mobility issues in wireless communications. As will be demonstrated in the present paper, it outperforms the other models in complex environments that possess many obstacles. At the same time, it respects the laws of motion, to permit a node to arrive at a predefined destination with the most convenient trajectory. That further allows mobile nodes to correctly address data packets to their destination through the most appropriate node trajectory even in the presence of a number of mobility constraints that must be satisfied. The results show that the proposed mobility model outperforms the existing models.

The remainder of this paper is organized as follows. Section 2 presents the related works of synthetic mobility models. Section 3 presents the implementation features of the proposed Maze Mobility Model. Section 4 shows the implementation process of MzMM. Section 5 offers the details MzMM movement properties. Section 6 analyses the MzMM network performance. And lastly, we present the main conclusions.

\section{Related works}

Many investigations on WSN have studied mobility models, and several synthetic mobility models have been suggested (Karamshuk et al. 2011; Da Cunha et al. 2014; Dong and Dargie 2013). As an alternative; in this section, we present an overview of the different mobility classes in order to set the background and the framework in which the proposed pattern has been developed, mainly for applications aimed at humans, animals, and vehicular motions. Mobility models are aimed at predicting the movement of nodes under different constraints. These synthetic models attempt to predict the trajectory of a node from its current location to the expected waypoint (destination) in accordance with its current direction, speed, and the time. With this purpose, these models are described by equations which take into account physical laws of motion. On the other hand, some models are inspired by real mobility traces in order to model real-life movements (Stegmann et al. 2018; Ibadah et al. 2018a). Notwithstanding, in all cases, the major challenge is how to achieve the best possible performance for wireless communications in the presence of frequent mobility (Batabyal and Bhaumik 2015). 
Synthetic mobility models are divided into four main categories: entity MMs (Dong and Dargie 2013), correlated or group-based MMs (Misra and Agarwal 2012), human or socially-based MMs (Pirozmand et al. 2014), and vehicular MMs (Ros et al. 2014). Each class has a specific mobility management which faces many challenges, including the selection of the appropriate topology. In the research described in this paper, the targeted class is the entity synthetic mobility model. This category allows each node to be independent of the others, i.e., each node independently selects its specific speed, pause time, and destination. Numerous entity synthetic mobility models have been proposed to deal with specific circumstances. Each pattern has a specific strategy according to a set of environmental parameters (random, spatial, historical, and geographical). These models are classified into the following sub-classes: random MMs, models with temporal dependence (temporal-based), models with spatial dependence (spatial-based), and models with geographic restrictions. Random mobility models are memoryless: each node is totally independent of the other nodes. These models randomly select a new waypoint without taking into consideration prior mobility decisions, such as the previous coordinates, speed, direction, and destination. Hence, these mobility models assume that mobile nodes can freely travel without any obstruction. These selections produce many mobility problems for these models, as pointed out in Panisson et al. (2012) and Ibadah et al. (2018b). In the case of models with temporal dependency, the devices are governed by physical laws of motion, where the current mobility decision mainly depends on the history of movements (Cong et al. 2015). Mobility models with spatial dependence estimate the future coordinates of the waypoint based on a probabilistic relation with the current location (Merkel et al. 2014). In models with geographic restrictions, the movement of the nodes neither are (often) random nor have temporal/spatial dependencies. However, the motion of a node is guided by paths, restricted in a building, or obstructed in a bounded area (Prabhakaran and Sankar 2006).

\section{Implementation features}

\subsection{The motivation for the Maze mobility model}

As previously introduced, many synthetic mobility models have been suggested. However, a challenge which must be faced is how, in complex environments to use these models to discover the most suitable trajectory to a specific destination. Some patterns imitate diverse real-life scenarios, but they are not adaptive with environment. Hence, they can't provide flexible mobility inside a complex area, and the performance is not improved.
In our daily life, two main mobility principles are employed: the 'discovery process', and 'moving with memory'. The 'discovery process' allows a physical searching at random for a given destination. This trivial procedure is on-demand. It is adopted by humans and animals for the first time to discover the correct path to a new location. Nevertheless, it has many weaknesses, given that it requires more time and has a high energy consumption during the search. On the other hand, the 'moving with memory' process avoids these defects by adopting a proactive strategy. It follows a likely-looking trajectory which has been already memorized. That is to say, if we are at home for the first time, we adopt a 'discovery process' but thereafter, we move from one room to another based on a 'remembering process', using the correct path without visiting all the rooms. Likewise, when we drive a car across a city with several streets and intersections, the pathway is chosen based on previous experiences, based on memory. Localization techniques like GPS have been deployed to assist driving, avoiding a random search, to choose the most suitable path to efficiently reach the desired destination. Therefore, the internal architecture of a house and its distribution of facilities, or the aerial view of a specific area, can be considered at first as a complex region which can be represented by a maze.

Inspired by real-life perceptions, the present paper proposes a realistic mobility model which is supported by a flexible path-discovery mechanism. The main goal of this investigation has been to find out how to provide mobile nodes with the ability to correctly move in the presence of diverse mobility constraints and obstructions (walls, intersections, and paths). As it is based on the principle of a discovery process to calculate the convient trajectory to a destination. Then, it saves it with the aim to allow mobile nodes moving based on memory. The proposed mobility model is called the Maze mobility model (MzMM).

\subsection{Algorithms for creating and solving mazes}

A maze is a kind of puzzle which is composed of a collection of paths and edges with known valid passages between two distant locations. Each complex region can be geometrically modeled as a specific maze. According to Foltin (2011), the maze features can be classified into seven classes: a maze is shaped by the combination of one characteristic from each class. These features usually influence the maze creation approach, which consequently impacts the solving strategy, including the required time, execution rate, and difficulty. All maze creation and maze solving algorithms are analyzed in Daedalus (Singh and Pandey 2014).

Several maze creation algorithms have been proposed to generate different shapes. An arbitrary maze geometry can be created to represent this model. In our implementation, we chose to generate a perfect maze (Kozlova et al. 2015a) due to 
its benefits which is characterized by an arbitrary set of closed circuits or loops, and has no inaccessible grid. An infinite grid of a perfect maze can be represented by a rectangular area with $m$ columns and $n$ rows constituting a set of $m \times n$ cells connected to each other by a unique path, where the total number of internal possible walls $W_{i}$ is defined by:

$W_{i}=2 \times m \times n-m-n$.

According to graph theory, a grid graph (Kersulis et al. 2018) is a subgraph of the infinite grid induced by a set of vertices. A rectangular $n \times m$ grid is the grid graph having the set of vertices $\left((X, Y) \in Z^{2} \mid 1 \leq x \leq n, 1 \leq y \leq m\right)$. It will be denoted by $G_{n}(m)$, as exactly shown in Fig. 1 .

Therefore, the Kirchoff theorem (Mohar et al. 1991) provides a systematic way to determine the number of possible spanning trees $t_{n}(m)$ of a given grid graph $G_{n}(m)$ using the adjacency matrix $\mathrm{A}(\mathrm{G})$.

$$
A(G)=\left(\begin{array}{ccccccc}
0 & 1 & 0 & \ldots & 0 & 0 & 0 \\
1 & 0 & 1 & \ldots & 0 & 0 & 0 \\
0 & 1 & 0 & \ldots & 0 & 0 & 0 \\
. & . & \ldots & & & . & . \\
. & . & . & & . & . & . \\
0 & 0 & 0 & \ldots & 0 & 1 & 0 \\
0 & 0 & 0 & \ldots & 1 & 0 & 1 \\
0 & 0 & 0 & \ldots & 0 & 1 & 0
\end{array}\right) .
$$

This method gives the number of polynomial trees of a specific grid graph without any a priori information of the construction of the trees. The adjacency matrix is not an appropriate tool, as it can't highlight the features of a grid structure. Furthermore, it doesn't provide any idea of the relations between the spanning trees. So, based on this matrix and using the Laplacien matrix $L=D-A$ where $D$ is the degree matrix (Mohar et al. 1991), the number of spanning trees $t_{n}(m)$ can be fixed on the basis of the grid structure. The number of spanning trees is deduced by using Eq. 2.

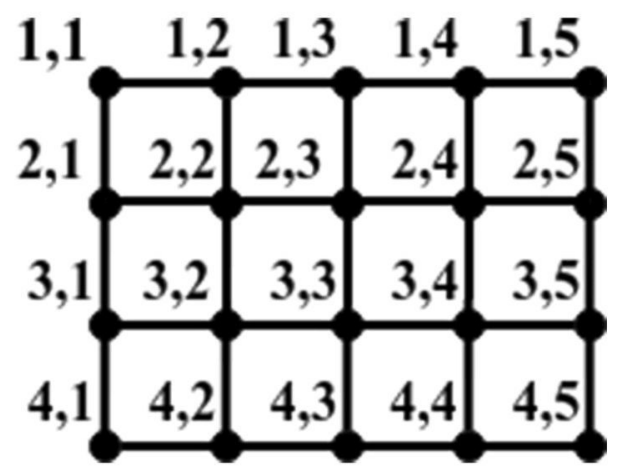

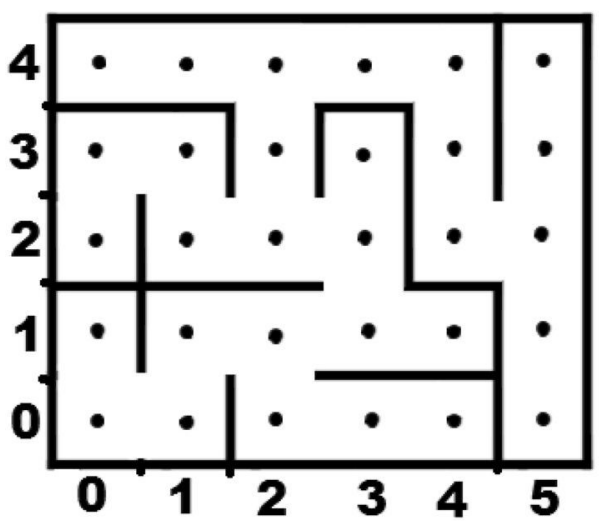

Fig. 2 Perfect maze generation

$t_{n}(m)=\frac{(2+\sqrt{3})^{m}-(2-\sqrt{3})^{m}}{2 \sqrt{3}}$.

The recursive division algorithm (Kozlova et al. 2015b) represents the most convenient algorithm to create this type of graph. It is the fastest algorithm with any directional biases. At the same time, it can arrange that multiple cells rival for binary tree mazes. These features eventually arrange that an undirected graph can depict the final perfect maze structure. Based on this process, the simulation area can be divided into black lines (walls) and white grids (passages), as shown in Fig. 2, where bold black lines represent walls.

According to Eq. (1), the number of walls inside a perfect maze is

$W_{p}=2 \times m \times n-m-n-(m \times n-1)=(m-1) \times(n-1)$.

Once the maze creation algorithm has successfully generated, the maze solving algorithm is executed in order to depict the final solved grid connections, as shown in Fig. 3a. So, to move from a source grid $\mathrm{xS}(\mathrm{i}), \mathrm{yS}(\mathrm{j})$ to a destination one $\mathrm{xD}(\mathrm{i}), \mathrm{yD}(\mathrm{j})$ without having any island (hole), an undirected graph as a binary tree arises from $t_{n}(m)$, as drawn in Fig. 3b. In our implementation, the recursive backtracker algorithm was adopted because it allows nodes a faster maze solving (Pech et al. 2015).

In Fig. 3, blue lines are the flight lights, and black points (round and squared) correspond to the grid centers, where square ones correspond to decision points. If a mobile node tries to travel from one location to another, the solving algorithm determines the correct path between these two selected points based on intermediate grids. For example, moving from $(0,5)$ to $(2,0)$, the resulting trajectory will be obvious as a fluent and realistic displacement due to the discovery

Fig. 1 Grid graph $G_{4}(5)$ 
Fig. 3 Perfect maze represented by an undirected graph. a Perfect maze solving and $\mathbf{b}$ undirected graph

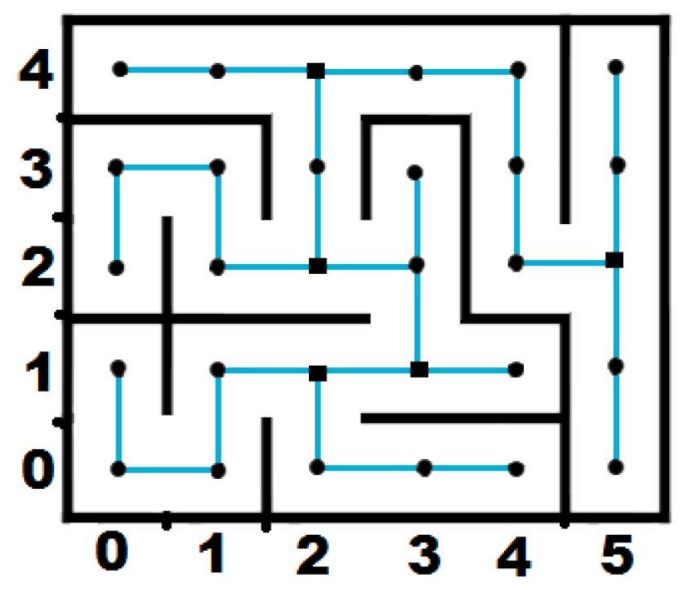

(a) Perfect maze solving

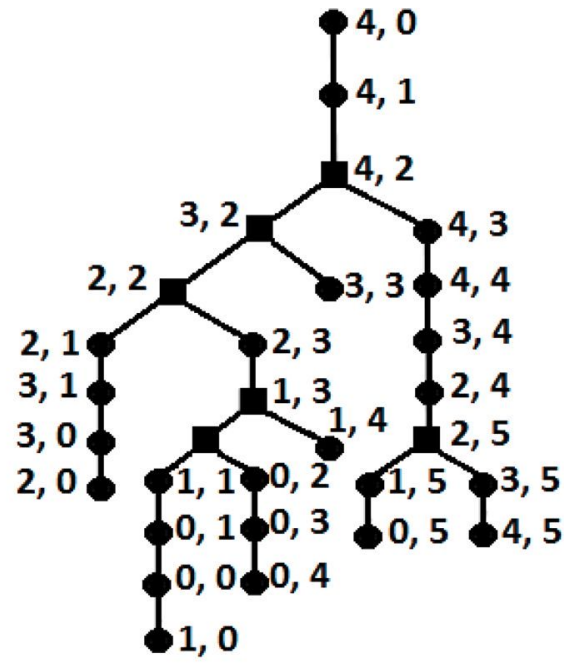

(b) Undirected graph of maze solving

Fig. 4 Correct path restitution based on: a perfect maze, $\mathbf{b}$ undirected graph

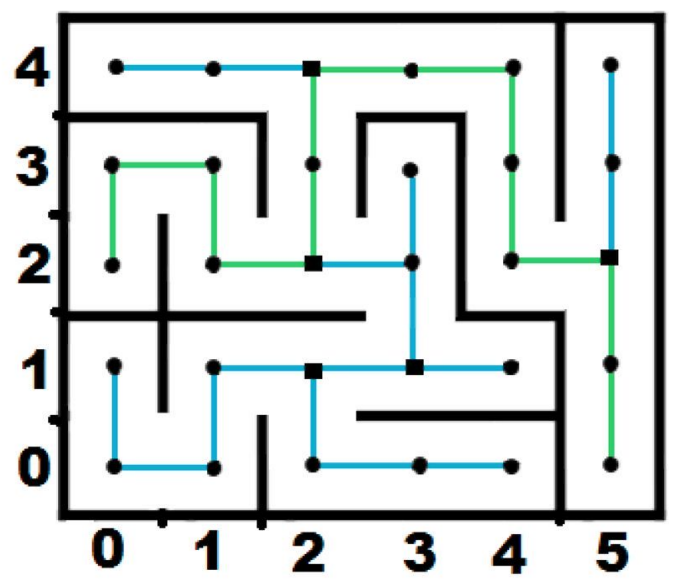

(a) Correct path between two different locations

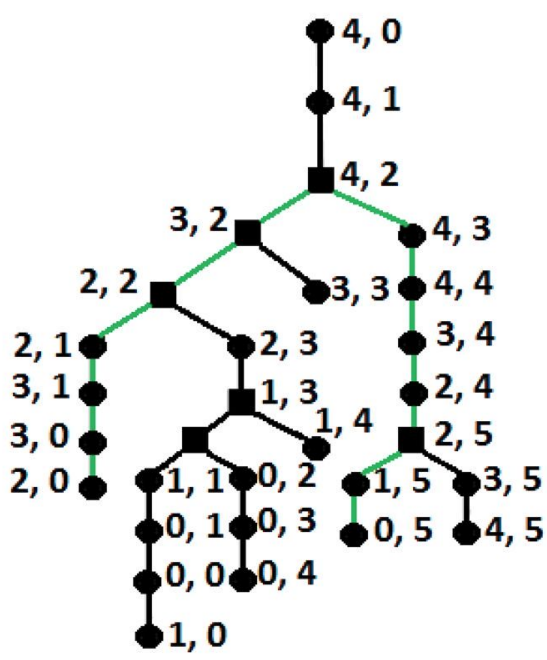

(b) (Path restitution based on the undirected graph mechanism process, as depicted in Fig. 4a, which is strongly related to the appropriate path provided by the undirected graph shown in Fig. 4b. From each decision point all possibilities are analysed (explored) to finally obtain the correct path which allow reaching the destination point. According to the solving algorithm, the resulting correct path can be described as follows: $(0,5)-(1,5)-(\mathbf{2}, \mathbf{5})-(2,4)-(3,4)-(4,4)-(4,3)-(\mathbf{4}, \mathbf{2})$ $(3,2)-(2,2)-(2,1)-(3,1)-(3,0)$, where the bolded points correspond to decision points. The detailed operation of MzMM is described in the next section.

\section{Implementation process of the Maze mobility model}

In our surroundings, we usually move according to existing geographic obstructions where our mobility behavior mainly depends on their particular locations. However, in a complex area where many obstacles can be found along the way, we can't successfully reach the actual position of our destination using the previously suggested synthetic mobility models: that requires a much more efficient model. This limitation prevents the use of the aforementioned patterns 


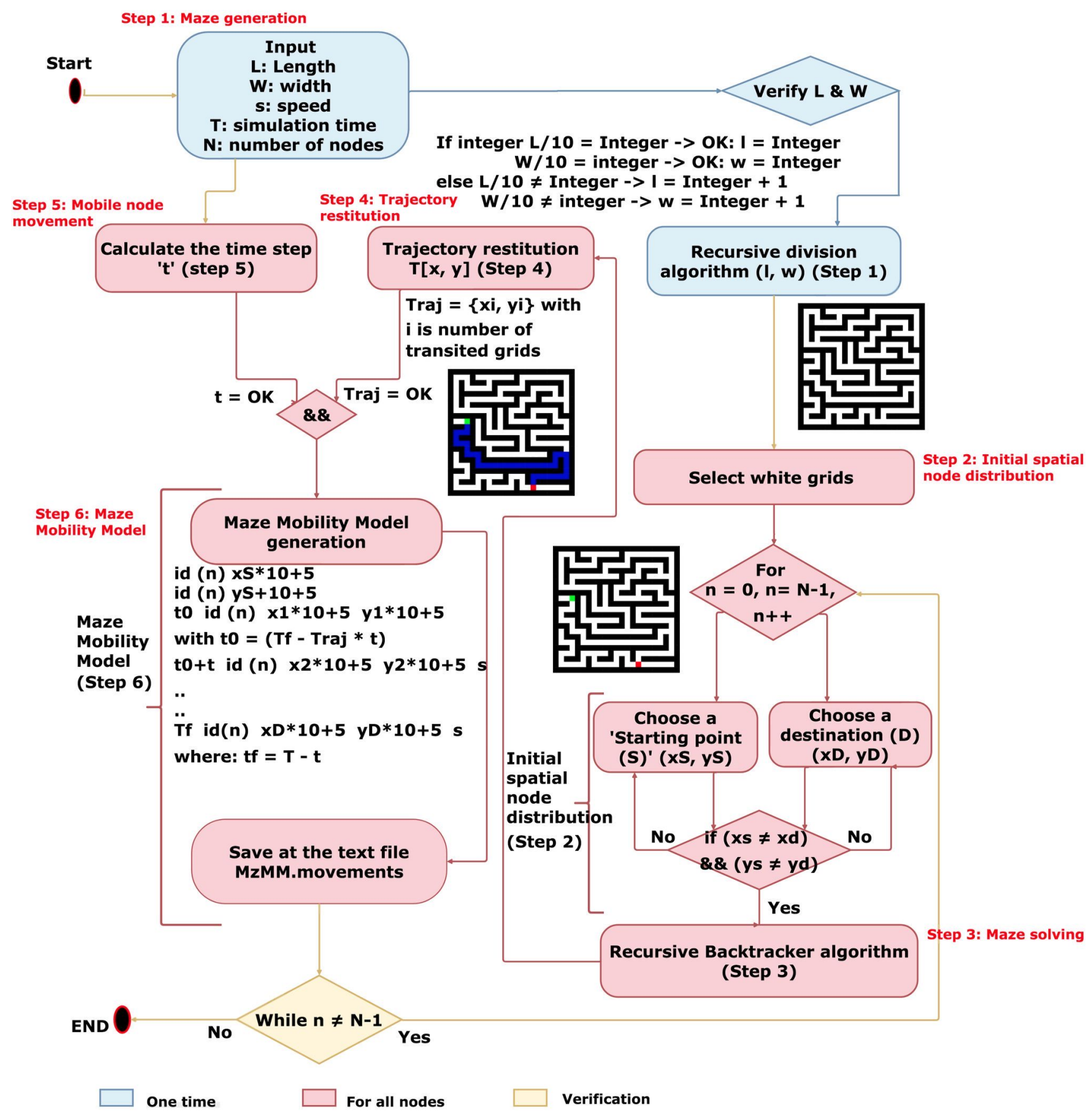

Fig. 5 Flow diagram of the MzMM implementation process

to take the correct motion decisions as a key feature even with realistic mobility constraints, as in the case of a maze. In such a case, a flexible mobility model is required, one that allows a mobile node to travel from one position to another one following the most suitable trajectory inside a specific maze. Therefore, in this investigation, we aim to itemize the proper steps of a new synthetic mobility model, which has been named the Maze Mobility Model. Figure 5 presents the flow diagram which describes the procedure to generate MzMM. The aforementioned steps are sequentially detailed in the following paragraphs.

Step 1 Maze generation

- The maze generation is the first step to implement the MzMM. Different shapes are available to generate a maze (Singh and Pandey 2014). In our case, we choose an $n \times n$ square or an $n \times m$ rectangular region to further evalu- 
ate network performance of the suggested model using a network simulator. Subsequently, a recursive division algorithm (Kozlova et al. 2015a) is used to create a maze which is divided into uniform grids as previously shown in Fig. 2 and implemented as described in Fig. 6 a.

For example, if we adopt grids of $10 \mathrm{~m}$ in an area of $100 \mathrm{~m} \times 100 \mathrm{~m}$, we obtain a uniform maze with 10 horizontal and 10 vertical grids.

- This procedure is generated one time for the whole duration of the experiment. The resulting design applied by the maze creation algorithm will be known by all mobile nodes in order to be able to solve it by using a maze solving algorithm (Step 3) before attempting to reach the destination through physical movements (Step 6).

Step 2 Initial spatial node distribution

- Before selecting the start and end locations by mobile nodes, the positions occupied by walls (black cells) are eliminated based on the maze generation procedure (Step 1 ), since in a realistic scenario moving from or to a wall is not feasible. Then, a starting point is randomly chosen from the available white grids, which is designated by the green color, and also an endpoint as a destination, designated by the red grid. These two positions must belong to two different positions of the white grids, as depicted in Fig. 6b.

\section{Step 3 Maze solving}

- The maze grids are divided into four categories: white grids, which represent passages; black grids, represent- ing walls; green grids, to designate the source position; and a red grid, to indicate the endpoint. A recursive backtracker algorithm (Pech et al. 2015) ensures the 'discovery process' before moving that takes into account all these grids to deduce the undirected graph of the created maze, as depicted in Fig. 3b. That correctly determines the trajectory from the starting point to the targeted destination, as shown in Fig. $6 \mathrm{c}$.

Step 4 Trajectory restitution

- According to the solving algorithm (Step 3), the positions of the grids visited are set on the maze. This process allows nodes to 'move with memory' which exactly forms the correct path from the source position to the destination, as represented by the green trajectory in Fig. 6b. This feature repeals mobile node to move searching the direction in such complex field. That saves time and conserves energy consumption for nodes.

\section{Step 5 Mobile node movement}

- Given the feedback provided by the maze-solving algorithm (Step 3), afterwards, the correct paths are constructed with positions $x$ and $y$ according to the accurate grids (Step 4). However, to make this model more realistic, it must respect the laws of motion, namely, $t=d / s$, with:

* $d$ represents the distance between two neighboring grids where each grid is identified by its center. Hence it makes $d=10 \mathrm{~m}$ due to the uniform maze distribution.
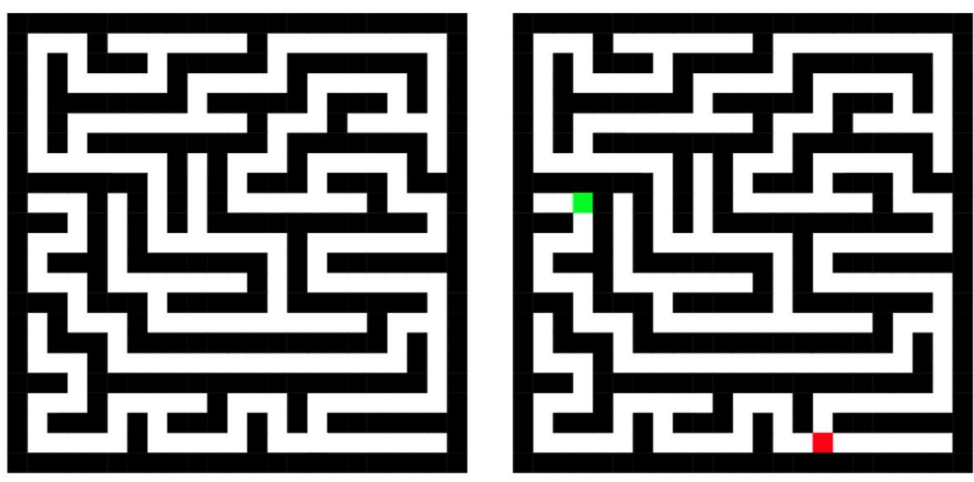

(a) Maze creation (Step 1) (b) Initial spatial node distribution (Step 2)

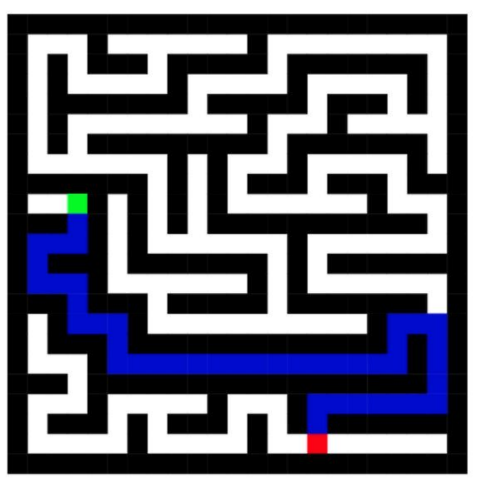

(c) Maze solving and trajectory restitution (Steps $3 \& 4$ )

Fig. 6 Maze mobility model implementation. a Maze creation, $\mathbf{b}$ initial spatial distribution of the nodes and $\mathbf{c}$ result of solving the maze 
* $s$ is the adopted speed during the execution of the algorithm. In our development and simulations, a constant speed of fast walking is assumed.

* Afterwards, $t$ is calculated according to the traveled distance and the selected speed.

Step 6 Maze mobility model

- From all the previously described steps, a coherent and realistic synthetic mobility model is obtained. The output of the MzMM contains all the information and features which describe the model.

\section{MzMM movement properties}

The mobility model proposed in this investigation possesses a number of basic features, as described in the next subsection which govern the whole adopted management strategy.

\subsection{Trajectory discovery}

Trajectory discovery is based on an algorithm which at every decision point along the path way analyses the different possibilities discarding those which get to no-exit points and considering those which grant further steps forward. For instance, the model resulting from Fig. 6c can be described in the following steps regarding the code parameters :

1. \$node_(0) set $X_{-} 105$

2. \$node_(0) set $Y_{-} 45$ :

- The first and the second lines represent setting, the positions of the starting point, which are selected from the initial spatial distribution (Step 2). In this case, the mobile node will be positioned at a given grid $(10,4)$ based on the previous set (according to Step 4).

- These values are multiplied by the grid distance $d$, which equals $10 \mathrm{~m}$, and added to $5 \mathrm{~m}$, which corresponds to the grid center reached (Step 5). So, the resulting $x$ and $y$ coordinates will be $x=(10 * 10+5)=105$ and $y=(4 * 10+5)=45$, as the final result to set real coordinates of the starting point in the aforementioned lines $\mathbf{1}$ and $\mathbf{2}$.

3. \$ns_at 0.0 \$node_(0) setdest 115455.0 :

- The third line shows the first grid after the starting point which belongs to the correct path (denoted in green) where: $t=0$, id_node $=0, x=115$ (i.e., $105+d), y=45$ and $s=5.0 \mathrm{~m} / \mathrm{s}$. In this example, after exploring the various possibilities and discarding the ones finishing at no-exit points, the mobile node moves down according to the given mobility decision, taking into consideration that it can only turn in one direction: left $(\mathrm{y}-\mathrm{d})$, up $(x-d)$, right $(y+d)$ or down $(x+d)$ depending on the result in Step 4.

4. \$ns_at 2.0 \$node_(0) setdest 125455.0

- The fourth line indicates the second grid after the starting point where $t=2.0 \mathrm{~s}$ (i.e., $d / \mathrm{s}$ ) and $x=125=(115+d)$ (i.e., the node moves down) with the same $y$ position.

In the next movement, a time lapse of $d / s$ is added to the last time $t$ (according to the temporal dependency approach described in Step 5).

The algorithm continues its execution for further lines follow up to the last one (nth line), the last decision point.

n. \$ns_at 88.0 \$node_( 0 ) setdest 2251655.0

Several mobility transitions are accomplished to finally reach the intended destination. The last line $n$ displays the positions $(x, y)$ of the last location, which is located at the $(22,16)$ grid. In this case, the node arrives at this position at time $t=d / s * 44$ where 44 represents the number of movement decisions between the source and the destination point. At the end, a decision tree with all the potential paths from the starting point is depicted, and the one getting the destination point with less steps (shortest way) in chosen as the discovered trajectory.

\subsection{MzMM features}

From all the above-mentioned steps, we conclude that MzMM is characterized by several remarkable features:

- MzMM respects the physical laws of motion: it takes into consideration the basic relations between distance, time, and speed (Step 5), unlike, for instance, the random model, which randomly selects the destination and speed independently of each other. That makes these pattern unrealistic.

- It can be considered a direction-history based model. It moves in one of the four possible directions (top, left, down, or right) mainly according to the last grid $(-d,+d)$. So, it is a spatially dependent mobility model. The next position can only be in one direction; left, up, right, or down (Step 4). 
- It is a time-dependent mobility model: the current time relies on previous time instants, i.e., basically $(t=d / s)$ (Step 5).

- The flight length is constant between two consecutive grids due to the uniform grid (Step 1).

- The return time and visiting frequency are probabilistically distributed, mainly in relation to the resulting trajectory (Steps 2 and 4 ).

- At the initial stage, it has a spatial distribution (white grids). In the subsequent stages, the resulting trajectory must be constituted only by passages without any walls, as shown by Fig. 6c. This fact imposes a realistic movement behavior that seems like that in daily-life.

The most significant movement features (compared to other mobility models) of the MzMM are highlighted in Table 1.

The meaning of the letters in Table 1 is as follows. C, constant; HB, history based; UD, uniformly distributed; PD, probabilistically distributed; P, periodic; PL, truncated power-law; UD, uniformly distributed; NA, not applicable. We conclude that the MzMM takes into consideration all the influential motion parameters so as to obtain a hybrid entity mobility model. It reflects a real-life context with several geographic constraints, e.g., walls (Step 1). MzMM permits a flexible motion even if in complex area, that can be shaped as a maze with diverse constraints. Based on the resulted model, mobile nodes can obtain the convient trajectory before moving (Step 5). The maze solving replaces the simple discovery process. And then, it allows generating a coherent mobility model which further will be followed by nodes while moving. It exactly reflects what is known as movement with memory. It is mainly inspired from human motion in real-life. It results in a flexible, accurate and robust mobility model which operate in complex areas, even when diverse mobility requirements are considered.
Based on the features of this approach shown above, we can conclude that it is more appropriate for supporting routing protocols in wireless networks. Furthermore, it can operate in complex areas showing a higher performance than other approaches developed so far, even when diverse mobility requirements are considered, as will be demonstrated in the next section.

\section{Performance analysis}

\subsection{Configuration parameters}

In this section, the performance of a given network when using the proposed mobility model (MzMM) is analyzed. The performance assessment was carried out as follows.

- The proposed MzMM has been compared with six wellknown mobility models, namely, two entity models: RandomWaypoint MM (RWMM) and ManhattanGrid MM (MGMM); two correlated models: Reference Point Group MM (RPGMM) and Nomadic MM (NMM); and two Human models: Self-Similar Least Action Walk (SLAW) and SMOOTH.

- Three mobile ad-hoc routing protocols are combined: one reactive protocol, Ad-hoc On-Demand Distance Vector (AODV); one proactive protocol, Optimized Link State Routing protocol (OLSR); and one Hybrid protocol, Zone Routing Protocol (ZRP).

- In order to analyze the impact of the simulation region, two different areas have been considered: a small one, $220 \mathrm{~m} \times 220 \mathrm{~m}$, and a large one, $1030 \mathrm{~m} \times 1030 \mathrm{~m}$.

- Three performance metrics are analyzed: Packet Delivery Ratio (PDR), average end-to-end delay, and network throughput.

Table 1 Movement features comparison: MzMM vs. other mobility models

\begin{tabular}{|c|c|c|c|c|c|c|c|}
\hline Mobility models & Model class & Speed & Direction & $\begin{array}{l}\text { Acceleration/ } \\
\text { deceleration }\end{array}$ & Pause time & Flight length & $\begin{array}{l}\text { Return time } \\
\text { and visiting } \\
\text { frequency }\end{array}$ \\
\hline MzMM Entity & Hybrid & $\mathrm{C}$ & HB & $\mathrm{C}$ & $\mathrm{C} / \mathrm{UD}$ & $\mathrm{C}$ & PD \\
\hline RWMM & Random (Entity) & UD & NA & NA & $\mathrm{C} / \mathrm{UD}$ & NA & NA \\
\hline MGMM & $\begin{array}{l}\text { Geographic } \\
\text { restrictions } \\
\text { (Entity) }\end{array}$ & $\mathrm{C} / \mathrm{UD}$ & UD on set of waypoints & NA & $\mathrm{C} / \mathrm{UD}$ & NA & NA \\
\hline RPGMM & Group/Correlated & $\begin{array}{l}\text { Based on } \\
\text { reference } \\
\text { point }\end{array}$ & Based on reference point & NA & $\mathrm{C} / \mathrm{UD}$ & NA & NA \\
\hline NMM & Group/Correlated & NA & Around reference point & NA & NA & NA & NA \\
\hline SLAW & Human & NA & PD on set destinations & NA & TPL & TPL & $\mathrm{P}$ \\
\hline SMOOTH & Human & NA & PD on set destinations & NA & TPL & TPL & $\mathrm{P}$ \\
\hline
\end{tabular}


Table 2 Simulation parameters

\begin{tabular}{ll}
\hline Parameters & Values \\
\hline Propagation model & TwoRayGround model \\
Bandwidth & $10 \mathrm{Mb} / \mathrm{s}$ \\
Number of nodes & 50 \\
Packet size & CBR \\
Packet rate & 512 bytes/s \\
Speed & $5 \mathrm{~m} / \mathrm{s}$ \\
Pause time (s) & 20 \\
Routing protocols & AODV \\
& OLSR \\
& ZRP \\
Mobility models & MzMM (Proposed model) \\
& RWMM, MGMM (Entity models) \\
& RPGMM, NMM (Correlated group MMs) \\
& SLAW, and SMOOTH (Human MMs) \\
Performance metrics & PDR \\
& Average e-e delay \\
& Throughput \\
Iterations & $220 \mathrm{~m} \times 220 \mathrm{~m}$ \\
& $1030 \mathrm{~m} \times 1030 \mathrm{~m}$ \\
& $1000 \mathrm{sec}$. \\
& 15 times \\
\hline
\end{tabular}

- The simulation results were obtained considering 42 different scenarios for the trials, 630 simulated files with an average of 15 iterations per scenario for $1000 \mathrm{~s}$.

The simulation parameters are combined to analyze all the possible cases in order to get a reliable and meaningful conclusion about the performance of the other mobility models versus the proposed MzMM. The experimental settings are presented in Table 2. The simulation results are described in Figs. 7, 8 and 9, and are discussed thereafter in Tables 3 and 4.

\section{Results and discussion}

Several performance metrics have been considered to correctly determine the performance of the synthetic mobility models to result in their behaviors when they are combined with other simulation features. On the other hand, the obtained outcomes are aimed at evaluating how MANET routing protocols influence their performance, as follows:

- Packet delivery ratio (PDR)

This metric provides the number of successfully delivered and received packets at a given destination compared to the total number of packets that have been sent out by the sender to the destination. When this metric reaches $100 \%$ means that all sent packets have been successfully received and the best performance has been achieved.

Based on an analysis of Fig. 7a, it can be observed that for the small simulation areas, for the PDR of MzMM, the result obviously outperforms the six other models for the three analyzed routing protocols AODV, OLSR, and ZRP, and this is given because the MzMM takes into account all the logical features. Furthermore for routing protocols, AODV shows the best outcomes due to its on-demand based operation. This leads the mobility model to show a proper performance where it exactly gets the correct path to reach a destination via route request RREQ and route reply RREP. The worst result is shown by ZRP, which must operate in coordination with Intra-zone Routing Protocol (IARP) and Inter-zone Routing Protocol (IERP) in order to explore the routing information. However, as shown in Fig. 7b, for large areas, the pattern proposed in this investigation achieves average outcomes for the three routing protocols, such as those shown by the human models (SLAW and SMOOTH). But the entity models obtain the worst results, due to their individual motion principle, the random mobility process of RWMM, and movements with geographic constraints in MGMM. It is worth noting that the correlated models obtain the best results when using the PDR protocol, thanks to the group strategy they use. However, the ZRP protocol excels by dint of splitting a spacious simulation area into small zones where it becomes easier to control the transmitted packets. for the three routing protocols, such as those shown by the human models (SLAW and SMOOTH). But the entity models obtain the worst results, due to their individual motion principle, the random mobility process of RWMM, and movements with geographic constraints in MGMM. It is worth noting that the correlated models obtain the best results when using the PDR protocol, thanks to the group strategy they use.

\section{- Average end-to-end delay}

This metric provides the time taken for a packet transmitted across the network to travel from its source node to its destination. A low value is considered a good end-to-end delay (ideally, zero seconds).

In the case of small areas, the results shown in Fig. 8a reveal that the MzMM approach outperforms the other models, obtaining results like those of the human models. It performs better than the entity and correlated models when using any of the three routing protocols considered in the investigation. Furthermore, the results shown in Fig. $8 \mathrm{~b}$ highlight that the proposed model surpasses the other ones when considering large areas, where it obtains 


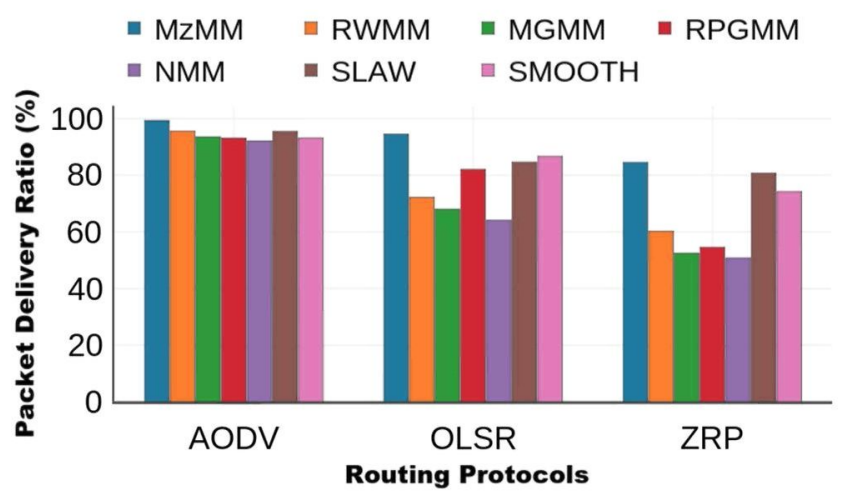

(a) Small area

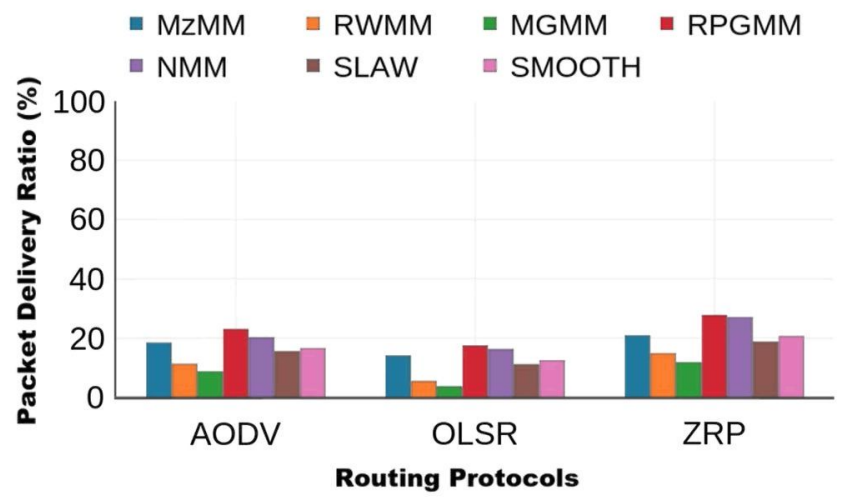

(b) Large area

Fig. 7 PDR (\%) of MzMM vs. other mobility models. a Small area, b large area

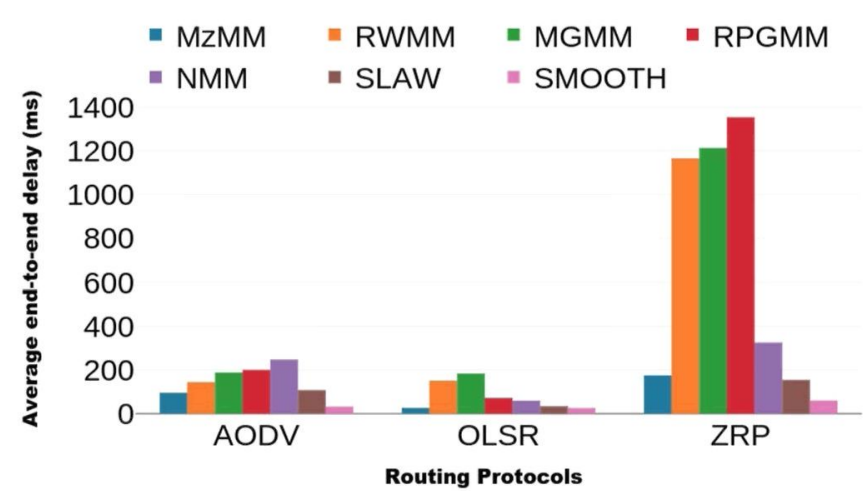

(a) Small area

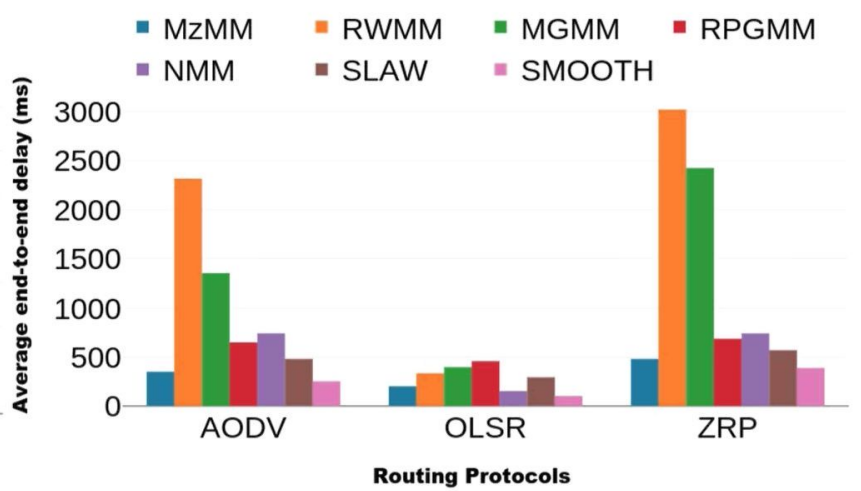

(b) Large area

Fig. 8 Average end-to-end delay (ms) of MzMM vs. other mobility models. a Small area, b large area

remarkable results compared to SMOOTH, RWMM, MGMM, RPGMM, and NMM, except the SMOOTH in this case. Moreover, the average end-to-end delay in the two considered areas obtain the best scores when using the proactive protocol OLSR. This result is obtained because the protocol always finds an available path to all destination since it searches routes in advance and continuously updates the required routing tables. On the other hand, ZRP is the protocol with the worst result in terms of this metric, on account of the long time it requires to locate a destination in a precise zone of the simulation region.

- Throughput

Network throughput refers to the rate of successfully delivered messages over a link; it is usually measured in bps (bits per second). The higher this value, the better the network performance. Figure 9a shows that in the case of a small area the MzMM model throughput is superior to that of the other tested models. Likewise, Fig. $9 \mathrm{~b}$ shows the robustness of the MzMM model which is better than those of all the other analyzed benchmark mobility models even when considering a wide simulation area.

After simulating 630 description files representing 42 different scenarios, the considered combinations mobility model-routing protocol have been scored and classified according to their results, which are summarized in Table 3. The best outcomes are shown in green (scores $1-3$ ), the acceptable results in yellow (score 4), and the worst results in red (scores 5-7).

After analyzing all the results shown above regarding the simulations performed under different conditions, we conclude that:

- MzMM outperforms the other mobility models considered in this investigation using different mobile ad-hoc routing protocols, such as AODV, OLSR, and ZRP.

- MzMM shows the best performance in terms of the three considered metrics PDR, average end-to-end delay, and throughput.

- MzMM is more stable even when various simulation areas are taken into account; small or large field. 


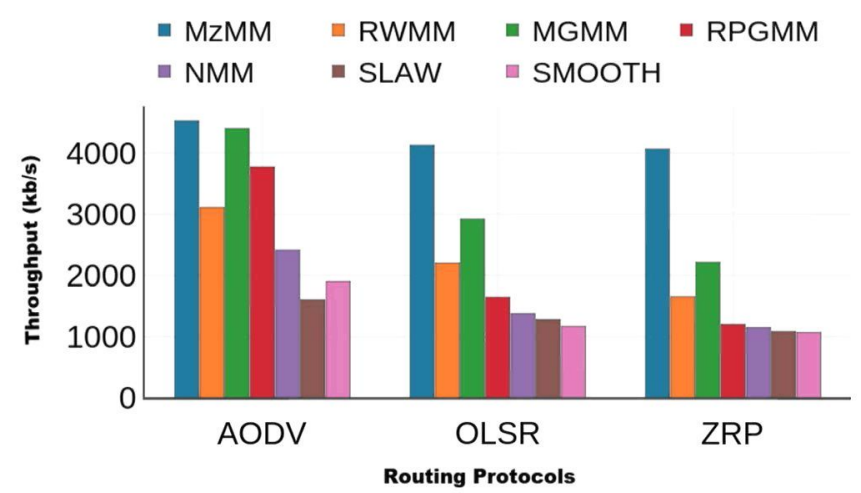

(a) Small area

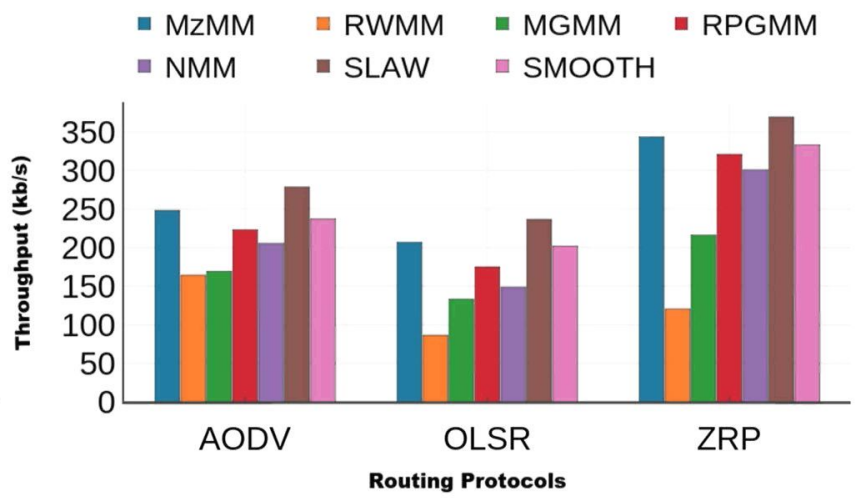

(b) Large area

Fig. 9 Throughput (kb/s) of MzMM vs. other mobility models. a Small area, b large area

Table 3 Experimental results summary

\begin{tabular}{|c|c|c|c|c|c|c|c|}
\hline \multirow{3}{*}{$\begin{array}{c}\text { Performance } \\
\text { Metrics }\end{array}$} & \multirow{3}{*}{ Mobility Models } & \multicolumn{6}{|c|}{ Routing protocols } \\
\hline & & \multicolumn{3}{|c|}{ Small area } & \multicolumn{3}{|c|}{ LARGE area } \\
\hline & & AODV & OLSR & $\overline{\mathrm{ZRP}}$ & AODV & OLSR & $\mathrm{ZRP}$ \\
\hline \multirow[t]{7}{*}{ PDR } & MzMM & 1 & 1 & 1 & 3 & 3 & 3 \\
\hline & RWMM & 3 & 5 & 4 & 6 & 6 & 6 \\
\hline & MGMM & 5 & 6 & 6 & 7 & 7 & 7 \\
\hline & RPGMM & 6 & 4 & 5 & 1 & 1 & 1 \\
\hline & NMM & 7 & 7 & 7 & 2 & 2 & 2 \\
\hline & SLAW & 2 & 3 & 2 & 5 & 5 & 5 \\
\hline & SMOOTH & 4 & 2 & 3 & 4 & 4 & 4 \\
\hline \multirow{7}{*}{$\begin{array}{c}\text { Avg } \\
\text { e-to-e delay }\end{array}$} & MzMM & 3 & 2 & 3 & 2 & 3 & 2 \\
\hline & RWMM & 4 & 6 & 5 & 7 & 5 & 7 \\
\hline & MGMM & 5 & 7 & 6 & 6 & 6 & 6 \\
\hline & RPGMM & 6 & 5 & 7 & 4 & 7 & 4 \\
\hline & NMM & 7 & 4 & 4 & 5 & 2 & 5 \\
\hline & SLAW & 2 & 3 & 2 & 3 & 4 & 3 \\
\hline & SMOOTH & 1 & 1 & 1 & 1 & 1 & 1 \\
\hline \multirow[t]{7}{*}{ Throughput } & MzMM & 1 & 1 & 1 & 2 & 2 & 2 \\
\hline & RWMM & 4 & 3 & 3 & 7 & 7 & 7 \\
\hline & MGMM & 2 & 2 & 2 & 6 & 6 & 6 \\
\hline & RPGMM & 3 & 4 & 4 & 4 & 4 & 4 \\
\hline & NMM & 5 & 5 & 5 & 5 & 5 & 5 \\
\hline & SLAW & 7 & 7 & 7 & 1 & 1 & 1 \\
\hline & SMOOTH & 6 & 6 & 6 & 3 & 3 & 3 \\
\hline
\end{tabular}

- MzMM is more efficient than the other mobility models in all combined scenarios, as shown in Table 3.

The remarkable results shown by the proposed mobility model (MzMM) have been obtained thanks to its conception, its logical process, the consideration of real-life movements, and all the features MzMM which have been previously detailed in Sect. 4. These remarks make the proposed pattern more stable, suitable, and efficient, even when in the presence of diverse mobility constraints. Table 4 summarizes and highlights the qualitative features of the validated mobility models, where $\mathrm{Y}$ means Yes, $\mathrm{N}$ means No, and A means Ambiguous.
Table 4 Qualitative features of mobility models

\begin{tabular}{lllll}
\hline $\begin{array}{l}\text { Mobility } \\
\text { models }\end{array}$ & Realistic & Scalable & $\begin{array}{l}\text { Possibility of a } \\
\text { mathematical } \\
\text { representation }\end{array}$ & $\begin{array}{l}\text { Uses } \\
\text { geographic } \\
\text { map }\end{array}$ \\
\hline MzMM & Y & Y & Y & Y \\
RWMM & N & Y & Y & N \\
MGMM & N & Y & N & N \\
RPGMM & N & Y & A & N \\
NMM & N & Y & N & N \\
SLAW & Y & N & A & Y \\
SMOOTH & N & Y & N & Y \\
\hline
\end{tabular}

$Y$ yes, $N$ no, $A$ ambiguous 


\section{Conclusion}

It is well known that the development of appropriate mobility models directly impacts the performance of wireless networks. In consequence, a proper understanding of the mobility features is relevant for designing and deploying outstanding mobile networks. Hence, network mobility models aim to characterize node movements inside a wireless network. In this paper, a new hybrid synthetic entity mobility model was proposed, which is called the Maze mobility model (MzMM). The new mobility model has been described and analyzed considering different sizes of the assessment region and using distinct routing protocols as well. The performance of MzMM has been compared with other well-known network mobility models, as RWMM,MGMM, RPGMM, NMM, SLAW, and SMOOTH by using typical network metrics: packet delivery ratio, average end-to-end delay, and network throughput. Based on the outcomes resulting from the implemented simulations. We deduce that the proposed MzMM offers a flexible mobility model more than previous approaches which allows adaptive movement even inside complex areas. This advantage has been achieved because the model is defined taking into account several real-life behaviors, such as temporal dependencies where current decisions are based on the timing of previous movements, as well as being based on spatial dependencies where the current position primarily depends on the last known location. Furthermore, it takes into considerations the geographic constraints defined inside the simulation area. The combination of these realistic features makes the MzMM more accurate and robust representing an hybrid entity mobility model par excellence, as supported by the high-performance results described in this paper. The simulation outcomes prove its robustness and efficiency. Future investigations will include verifying its actual accuracy depending on diverse mobility problems, such as the speed decay problem, the spatial distribution of the nodes, the density wave phenomenon, and the mobile neighbors range.

\section{References}

Batabyal S, Bhaumik P (2012) Improving network performance with affinity based mobility model in opportunistic network. In: Wireless telecommunications symposium (WTS), IEEE, pp 1-7

Batabyal S, Bhaumik P (2015) Mobility models, traces and impact of mobility on opportunistic routing algorithms: a survey. IEEE Commun Surv Tutor 17(3):1679-1707

Cheng X, Huang X, Du D-Z (2013) Ad hoc wireless networking, vol 14. Springer Science \& Business Media, Berlin

Cong Y, Zhou X, Kennedy RA (2015) Interference prediction in mobile ad hoc networks with a general mobility model. IEEE Trans Wirel Commun 14(8):4277-4290
Conti M, Giordano S (2014) Mobile ad hoc networking: milestones, challenges, and new research directions. IEEE Commun Mag 52(1):85-96

Da Cunha FD, Boukerche A, Villas L, Viana AC, Loureiro AA (2014) Data communication in VANETs: a survey, challenges and applications. PhD thesis, INRIA Saclay; INRIA

Dong Q, Dargie W (2013) A survey on mobility and mobility-aware mac protocols in wireless sensor networks. IEEE Commun Surv Tutor 15(1):88-100

Foltin M (2011) Automated maze generation and human interaction. Masaryk University Faculty of Informatics, Brno

Ibadah N, Minaoui K, Rziza M, Oumsis M (2017) Experimental synthesis of routing protocols and synthetic mobility modeling for MANET. In: International conference on sensor networks, vol 2. SciTePress, pp 168-173

Ibadah N, Minaoui K, Rziza M, Oumsis M, Benavente-Peces C (2018a) Smart collection of real-time vehicular mobility traces. Future Internet 10(8):78

Ibadah N, Minaoui K, Rziza M, Oumsis M, Benavente-Peces C (2018b) Deep validation of spatial temporal features of synthetic mobility models. Computers 7(4):71

Karamshuk D, Boldrini C, Conti M, Passarella A (2011) Human mobility models for opportunistic networks. IEEE Commun Mag 49(12): 157-165

Kersulis JA, Hiskens IA, Coffrin C, Molzahn DK (2018) Topological graph metrics for detecting grid anomalies and improving algorithms. In: 2018 Power systems computation conference (PSCC), IEEE, pp 1-7

Kozlova A, Brown JA, Reading E (2015a) Examination of representational expression in maze generation algorithms. In: 2015 IEEE conference on computational intelligence and games (CIG), IEEE, pp 532-533

Kozlova A, Brown JA, Reading E (2015b) Examination of representational expression in maze generation algorithms. In: Computational intelligence and games (CIG), 2015 IEEE Conference on IEEE, pp 532-533

Merkel S, Mostaghim S, Schmeck H (2014) Hop count based distance estimation in mobile ad hoc networks-challenges and consequences. Ad Hoc Netw 15:39-52

Misra S, Agarwal P (2012) Bio-inspired group mobility model for mobile ad hoc networks based on bird-flocking behavior. Soft Comput 16(3):437-450

Mohar B, Alavi Y, Chartrand G, Oellermann O (1991) The laplacian spectrum of graphs. Graph Theory Comb Appl 2(871-898):12

Panisson A, Barrat A, Cattuto C, Van Den Broeck W, Ruffo G, Schifanella R (2012) On the dynamics of human proximity for data diffusion in ad-hoc networks. Ad Hoc Netw 10(8):1532-1543

Pech A, Hingston P, Masek M, Lam CP (2015) Evolving cellular automata for maze generation. In: Australasian conference on artificial life and computational intelligence, pp 112-124, Springer

Pirozmand P, Wu G, Jedari B, Xia F (2014) Human mobility in opportunistic networks: characteristics, models and prediction methods. J Netw Comput Appl 42:45-58

Prabhakaran P, Sankar R (2006) Impact of realistic mobility models on wireless networks performance. In: Wireless and mobile computing, networking and communications, 2006. (WiMob'2006). IEEE International Conference on IEEE, pp 329-334

Ros FJ, Martinez JA, Ruiz PM (2014) A survey on modeling and simulation of vehicular networks: communications, mobility, and tools. Comput Commun 43:1-15

Singh B, Pandey AK (2014) Maze using hybrid genetic algorithm. In: International conference of advance research and innovation (ICARI-2014), pp212-216

Stegmann RA, Žliobaitè I, Tolvanen T, Hollmén J, Read J (2018) A survey of evaluation methods for personal route and destination 
prediction from mobility traces. Wiley Interdiscipl Rev Data Min Knowl Discov 8(2):e1237

Tomasini M, Mahmood B, Zambonelli F, Brayner A, Menezes R (2017) On the effect of human mobility to the design of metropolitan mobile opportunistic networks of sensors. Pervasive Mob Comput 38:215-232 